\title{
Article
}

\section{Aeroelastic Stability of Combined Plunge-Pitch Mode Shapes in a Linear Compressor Cascade ${ }^{\dagger}$}

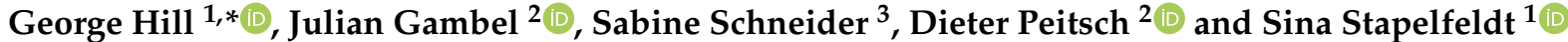 \\ 1 Rolls-Royce Vibration UTC, Imperial College London, London SW7 2AZ, UK; s.stapelfeldt@imperial.ac.uk \\ 2 Chair of Aero Engines, TU Berlin, 10587 Berlin, Germany; julian.gambel@tu-berlin.de (J.G.); \\ dieter.peitsch@tu-berlin.de (D.P.) \\ 3 Rolls-Royce Deutschland Ltd. \& Co. KG, Dahlewitz, 15827 Blankenfelde-Mahlow, Germany; \\ Sabine.Schneider@Rolls-Royce.com \\ * Correspondence: g.hill18@imperial.ac.uk \\ + This paper is an extended version of our paper published in the Proceedings of the 14th European \\ Turbomachinery Conference, Gdansk, Poland, 12-16 April 2021.
}

\begin{abstract}
Modern aeroengine designs strive for peak specific fuel and thermal efficiency. To achieve these goals, engines have more highly loaded compressor stages, thinner aerofoils, and blended titanium integrated disks (blisks) to reduce weight. These configurations promote the occurrence of aeroelastic phenomena such as flutter. Two important parameters known to influence flutter stability are the reduced frequency and the ratio of plunge and pitch components in a combined flap mode shape. These are used as design criteria in the engine development process. However, the limit of these criteria is not fully understood. The following research aims to bridge the gap between semi-analytical models and modern compressors by systematically investigating the flutter stability of a linear compressor cascade. This paper introduces the plunge-to-pitch incidence ratio, which is defined as a function of reduced frequency and pitch axis setback for a first flap (1F) mode shape. Using numerical simulations, in addition to experimental validation, aerodynamic damping is computed for many modes to build stability maps. The results confirm the importance of these two parameters in compressor aeroelastic stability as well as demonstrate the significance of the plunge-to-pitch incidence ratio for predicting the flutter limit.
\end{abstract}

Schneider, S.; Peitsch, D.; Stapelfeldt,

S. Aeroelastic Stability of Combined

Plunge-Pitch Mode Shapes in a

Linear Compressor Cascade. Int. J.

Turbomach. Propuls. Power 2022, 7, 7 .

https://doi.org/10.3390/

ijtpp7010007

Received: 8 December 2021

Accepted: 8 February 2022

Published: 14 February 2022

Publisher's Note: MDPI stays neutral with regard to jurisdictional claims in published maps and institutional affiliations.

Copyright: (c) 2022 by the authors. Licensee MDPI, Basel, Switzerland. This article is an open access article distributed under the terms and conditions of the Creative Commons Attribution (CC BY-NC-ND) license (https://creativecommons.org/ licenses/by-nc-nd/4.0/)

\section{Introduction}

Modern aeroengine compressor designs strive to reduce weight and maintain peak efficiency. The implementation of new features to achieve these goals pose issues for compressor aeroelastic stability. For example, weight can be reduced and efficiency increased by designing thinner aerofoils; however, these have greatly reduced stiffness. Similarly, titanium blade-integrated disks (blisks) reduce weight but also decrease structural damping gained by hub-blade contacts. This reduction in damping is accompanied by a trend of increased loading per stage, to either increase the overall pressure ratio or maintain the pressure ratio with a reduced number of stages. These design trends make the blades more susceptible to experiencing high vibration amplitudes due to flutter. Flutter is an aeroelastic instability in which energy is transferred from the flow into a mechanical structure, leading to exponentially growing vibration amplitudes. Close to the stall boundary at part speed operating conditions, it is often flutter (rather than stall or surge) that represents the limiting condition for safe engine operation. For this reason, the identification of the key flutter parameters-and a prediction of the stability limit-is essential during early engine design processes in order to avoid costly re-designs later on. Clearly, one of the key influences on compressor flutter stability is the frequency and mode shape of vibration. Generally, increasing the reduced frequency improves flutter stability for a fixed mode shape. A mode 
shape consists of both a blade (e.g., flap, torsion, bending) and disk assembly (nodal diameter) mode. This paper will investigate the stability in the first flap (1F) blade mode. Since most of the aeroelastic work input occurs at spanwise sections near the tip of the blade, it will consider the stability of a quasi two-dimensional blade section. It assumes that a spanwise section of the 3D flap mode can be represented by a two-dimensional rigid body motion comprising a plunging and pitching component, as illustrated in Figure 1, where dashed lines represent the vibration displacement. This assumption is valid as long as the flexion of the blade itself is small compared to the rigid motion. The mode shape composition is controlled by the position of the pitch axis, with the plunge component increasing the further aft the pitch axis is located.

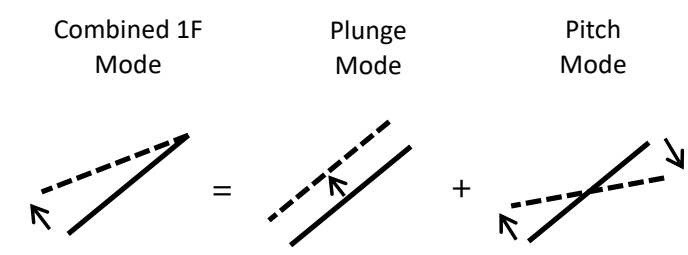

Figure 1. The 1F mode shape as the superposition of plunge and pitch components.

It is well-known that the proportion of the bending and torsion (or the ratio of plunge and pitch in 2D) components in the first flap mode is an important parameter for flutter. The importance of this parameter has been demonstrated in a number of studies, ranging from flat plates with zero mean deflection [1] to fans [2,3] and turbines [4]. All of these consider modes where the plunge and pitch motion are in phase. The aerodynamic damping, which is negative when flutter is produced, in the first flap mode can be conceptually split into four terms: (1) plunge-induced damping in the plunge mode, (2) the plunge-induced damping in the pitch mode, (3) the pitch-induced damping in the plunge mode, and (4) the pitch-induced damping in the pitch mode. While the pure plunge component (1) is often stable and the pure pitch component (4) is small in magnitude, the cross-coupling terms (2)(3) have been identified as the most important contributors to instability [2,3,5]. Under finite loading, blades can become unstable in a pure plunge mode [6], and therefore the pure plunge component cannot be considered unconditionally stable.

Steady loading, in addition to reduced frequency and mode shape composition, is an important factor in flutter stability. This applies to cascades, where it has been shown that the critical reduced frequency increases with increasing flow deflection for a fixed mode shape [6], as well as to rotors, where aerodynamic damping decreases with a reduction in mass flow rate. Although the trend with steady loading is clear, the interaction with the other parameters is not completely understood, and a detailed discussion is out of the scope of this paper. The reader is referred to the literature such as [6] for a systematic exploration of blade loading in a compressor cascade, and [3] for a discussion in the context of modern fan blades.

Neglecting the effects of steady loading, plots of aerodynamic damping against pitch axis location and reduced frequency are often employed to map stability $[1,4,7]$. When applied to 3D blades, this is usually measured at the tip section of the blade, for example, see [8]. The stability boundary then follows a curved line, as sketched in Figure 2. To combine both reduced frequency and mode shape composition into a single parameter, an incidence ratio $\phi=\theta_{\text {plunge }} / \theta_{\text {pitch }}$ can be defined, where $\theta_{\text {plunge }}$ and $\theta_{\text {pitch }}$ are the quasisteady incidence induced by the plunge and pitch components, respectively. Exactly how $\phi$ relates to pitch axis location and reduced frequency will be shown in the next section. Experience on highly loaded transonic fan blades, as reported in $[3,7,8]$, suggests that the stability limit lies at $\phi \approx 1$.

The validity of the incidence ratio $\phi$ as a flutter stability parameter has not been demonstrated in the public domain. This paper will confirm its importance using a joint 
computational and experimental test campaign on a linear compressor cascade, specifically designed for this purpose. The computational fluid dynamics (CFD) is validated against measurements, and aerodynamic damping is computed for a wide range of pitch axis locations and reduced frequencies. The stability boundary is then determined in terms of the plunge-to-twist incidence ratio.

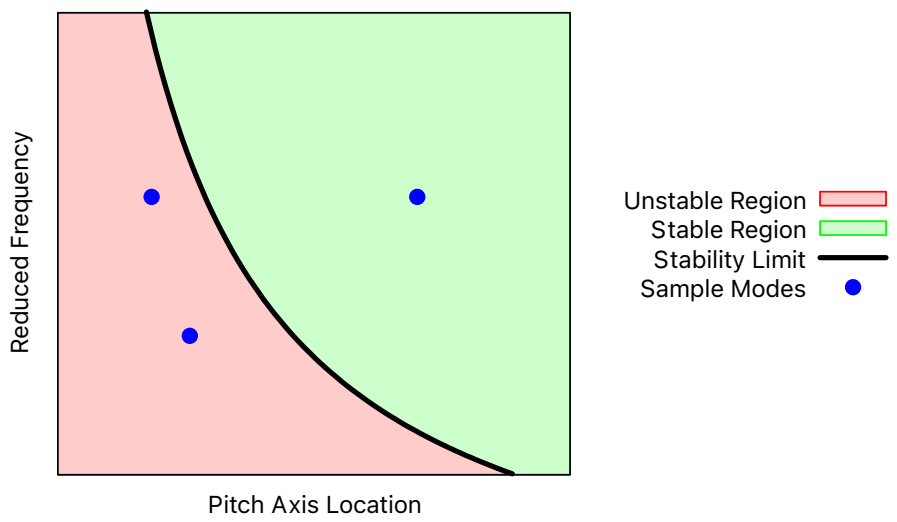

Figure 2. Sketch of stability map.

\section{Test Case}

As mentioned, this work focuses on a two-dimensional 1F blade mode shape, which can be considered a superposition of pure plunge and pure pitch components. Under the assumption that these components are in phase, and that the blades are rigid bodies, the mode shape is equivalent to a single degree of freedom (SDOF) rotation about a pitching axis located a chordwise distance $x$ downstream of the leading edge. An implementation of this is shown in Figure 3.

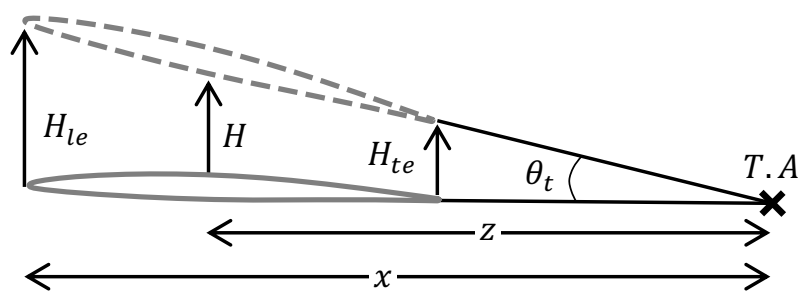

Figure 3. Implementation of $1 \mathrm{~F}$ mode shape as SDOF rotation.

By changing the positions of the pitching axis location $x$ and the mid-chord plunge amplitude $H$, the plunge-to-twist ratio of the mode can be varied. The limits are a pure twist mode when $x=0.5 c$ and a pure bending mode as $x \rightarrow \infty$. We will now define the the plunge-to-twist incidence ratio $\phi$ as the ratio of incidences generated by each of the components' vibration amplitude. The pitching axis location, $x$, relates to the plunge-totwist incidence ratio as follows:

$$
\phi=\frac{\theta_{p}}{\theta_{t}}=\frac{(\omega H / u)}{(H / z)}=\frac{\omega z}{u}=k\left(\frac{x}{c}-\frac{1}{2}\right)
$$

where $k=\frac{\omega c}{u}$ is the reduced frequency and $z=x-c / 2$ is the experimental lever length. From Equation (1), we can see that properties of the mode shape can be varied as a function of the normalised pitching axis location $x / c$ and the reduced frequency $k$, whereas $\phi$ is the derived stability parameter which will form the basis of the stability map. Due to experimental constraints, the twist incidence amplitude, $\theta_{t}$, is fixed at $1.5^{\circ}$.

Investigations into aeroelastic stability were conducted on a subsonic, linear compressor cascade. The blade profiles are the modified NACA 65-Series [9], with a maximum 
thickness of $6 \%$ at half-chord over a $10^{\circ}$ circular arc camber line. The stagger is fixed at $43^{\circ}$, with a span-to-chord ratio of 1.313 . The cascade is formed by an 11-blade passage, with a pitch-to-chord ratio, $s / c$, of 0.75 , which aims to represent a section cut of a typical compressor blade in the tip region. The flow incidence is fixed at $2^{\circ}$, and the operating point is far away from stall. The cascade has viscous hard walls on the hub, tip, and blade-to-blade boundaries, as shown in Figure 4. Inflow and outflow boundary conditions are highlighted in green, while all other viscous hard walls are shown in black.

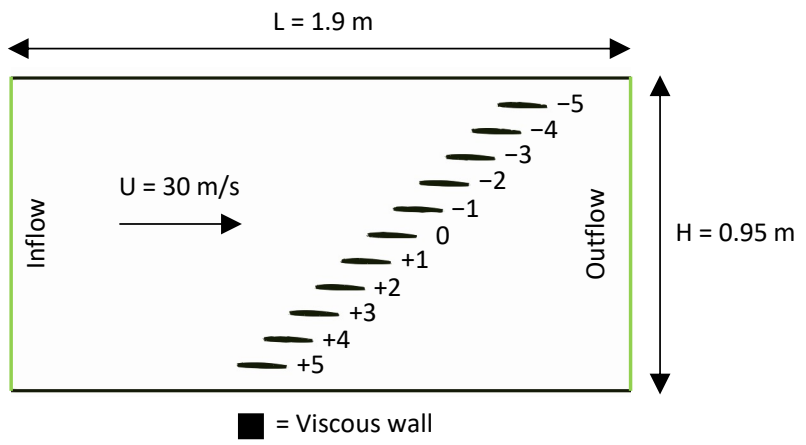

Figure 4. Schematic of cascade geometry with blade numbering convention.

The central Blade 0 will vibrate with a prescribed motion, and an aerodynamic influence coefficient method (AIC) will be used to calculate the aerodynamic damping ratio for many assembly modes. All other blades are fixed. The amplitude is large enough for unsteady pressures to be distinct from numerical noise but small enough for the unsteady flow to be represented as linear. Nominal operating conditions and parameter ranges are listed in Table 1. Both the reduced frequency and the normalised pitching axis location are varied with constant $5 \%$ increments between the limits shown. The reduced frequency range corresponds to a physical frequency range of 6.36-22.28 Hz.

Table 1. Cascade operating conditions.

$\begin{array}{lc}\text { Mach number (-) } & 0.087 \\ \text { Reynolds number (-) } & 300,000 \\ \text { Incidence angle (deg) } & 2 \\ \text { Reduced frequency range (-) } & 0.2-0.7 \\ \text { Normalised torsion axis location (-) } & 0.5-3\end{array}$

\section{Test Rig}

The experimental investigations were conducted at the Chair for Aero Engines, TU Berlin. The wind tunnel and cascade architecture are shown in Figure 5. The air flow is provided by a radial blower, which is then homogenised by passing through a diffuser and a settling chamber with multiple honeycomb grids and a nozzle before entering the measurement section. The air is discharged as a free jet at the end of the measurement section. Total pressure and temperature are measured at the settling chamber side wall, dynamic pressure is measured with a Pitot-static probe at the centre of the measurement section inlet face, and inlet static pressure is measured at the inlet face side wall. The mass flow rate and Reynolds number are calculated. Time-averaged and turbulent velocity distributions at the measurement section inlet as well as flow patterns can be found in [10]. 


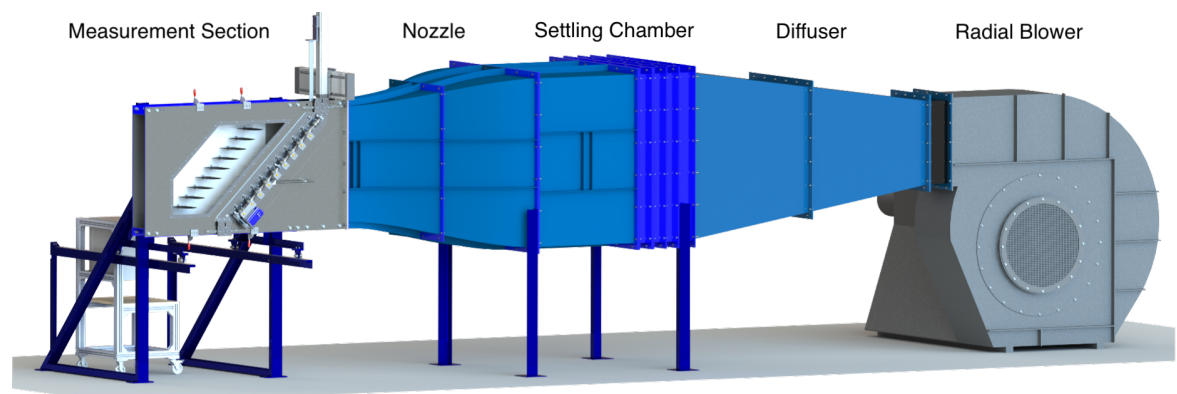

Figure 5. Experimental test rig architecture.

The central blade of the cascade is attached at its root to a lever on the outside of the wind tunnel. The lever is mounted to a traversing plate, which can be moved back and forth along linear bearings on the side wall to change the pitching axis location. Figure 6 shows a section cut of the traversing plate at its maximum x/c. Moving the blade position in the slot fixes the location in the cascade. Oscillatory motion is provided by an electric step motor, with an eccentric attached to its shaft through a scotch yoke mechanism and a connecting rod. Lever displacement is monitored with a laser triangulation distance sensor at the tip of the lever.

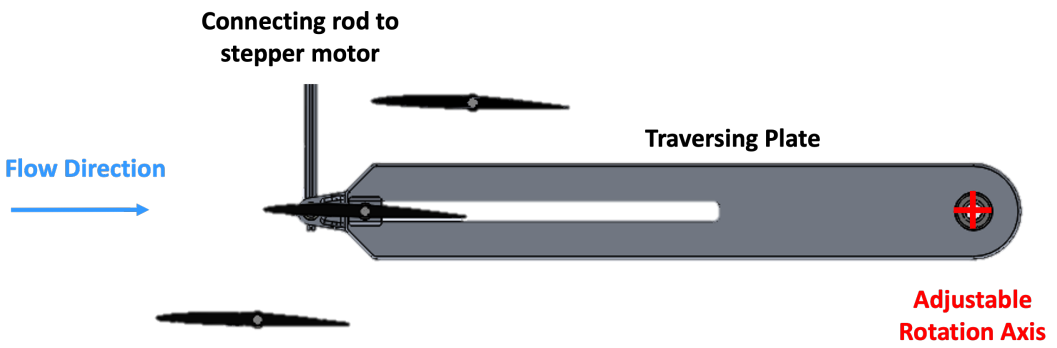

Figure 6. Section view of the vibrating blade module implementation of $x / c$.

Piezo-resistive differential pressure transducers were employed to measure the blade surface pressures. The pressure transducers exhibited linear behaviour around the zero point and can be considered as bidirectional. Calibration was conducted against externally calibrated precision pressure cells, which showed accurate pressure readings in the singledigit $\mathrm{Pa}$ range. The data acquisition system provided a theoretical resolution of $0.0267 \mathrm{~Pa}$. The measurement blades have mid-span 20 pressure taps, with 10 each for the suction and pressure surface. The pressure taps have an exponential spacing along the chord, with greater density towards the leading edge. Transition strips are located between taps 4 and $5(19 \%$ chord $)$ on the suction surface of all blades to prevent flow separation.

\section{Numerical Method}

\subsection{Flow Solver}

The solver implemented throughout this work, AU3D, has been developed and validated at Imperial College over the last 25 years [11-13]. It is a 3D, time-accurate, viscous, finite-volume unsteady Reynolds averaged Navier-Stokes (URANS) solver and is secondorder accurate in time and space. An edge-based data structure is used in the solver for computational efficiency. Turbulent closure is provided by a modified one-equation Spalart-Allmaras turbulence model [14]. The scheme implements a viscous wall function to model the flow near the wall. Steady state solutions are obtained using a RANS method, with a dual time-stepping procedure and residual smoothing to accelerate convergence. The mesh movement is satisfied by a spring analogy algorithm [11]. 


\subsection{Computational Implementation}

The computational mesh is semi-unstructured. In the spanwise direction, the domain is discretised by 23 radial layers. All viscous surfaces (including hub, tip, and spanwise boundaries) have a fully resolved boundary layer as shown in the upper wall mesh in Figure 7a. The boundary layer is resolved with a high density of hexahedral elements, with an average of $y+=45$ required by the viscous wall functions. A typical blade surface boundary layer is shown in Figure $7 \mathrm{~b}$. A total of $98 \%$ of the surface elements have $\{20<y+<100\}$. The far field contains a coarser prismatic mesh and Riemann invariant boundary conditions at the inflow and outflow. This minimises the undesirable reflection of disturbances off the boundaries, which may impact flutter stability. The inlet boundary is placed approximately 4 chords upstream of the first blade. The overall mesh contains approximately $1.7 \times 10^{6}$ nodes. This mesh was selected based on agreement with the experimental data.

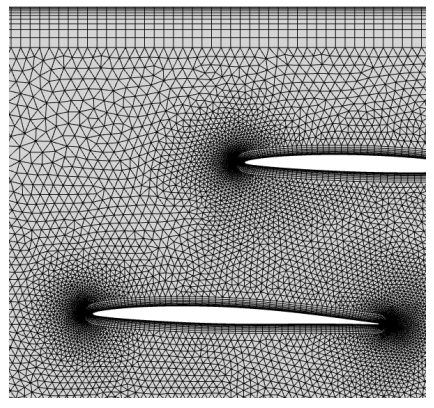

(a) Mesh of upper wall.

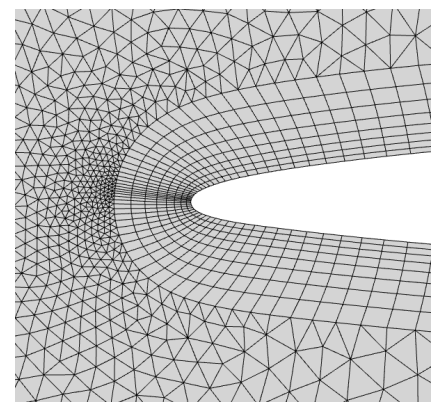

(b) Blade leading edge mesh.

Figure 7. Linear cascade mesh.

The steady state flow solutions are obtained by imposing total pressure, total temperature, and flow angle at the far-field inflow boundary as well as static pressure at the outflow boundary. The mode shape is obtained analytically by the axis rotation of each surface node about the torsion axis location, as in the experiment. The mode is then mass normalised and the vibration amplitude is satisfied by the modal displacement. The blade vibrates with the prescribed motion and is discretised in time, with approximately 350 time steps per vibration cycle, based on a time convergence study. To match the experimental work, the modal force is calculated using only the mid-span unsteady pressures and assumes a radially uniform flow.

\section{Aero-Damping Evaluation Method}

The flutter stability of the cascade is assessed by an aerodynamic influence coefficient (AIC) method [15]. This treats the modal force on the blade surface as the linear sum of influences of the vibrating blade acting on itself and the influence of propagating disturbances from the adjacent blades' vibrations. Clearly, the propagation of disturbances that will influence a given blade will be affected by the geometry, operating conditions, and mode shape. The influence coefficients form a circulant matrix $L$ defined as follows:

$$
f_{i}(t)=\left[\begin{array}{ccccc}
L_{0} & L_{N-1} & L_{N-2} & \cdots & L_{1} \\
L_{1} & L_{0} & L_{N-1} & \cdots & L_{2} \\
\vdots & \vdots & \vdots & & \vdots \\
L_{N-1} & L_{N-2} & L_{N-3} & \cdots & L_{0}
\end{array}\right]\left\{\begin{array}{c}
q_{0}(t) \\
\vdots \\
q_{i}(t) \\
\vdots \\
q_{N-1}(t)
\end{array}\right\}
$$

where $q$ is the modal displacement, $f$ is the modal force, $L$ is the complex influence coefficient, and $N$ is the number of blades. The dominant terms of the $L$ matrix are the diagonal $L_{0}$ terms, which represent the modal force influence on a blade due to its own 
vibration, which always provides a stabilising contribution [16]. The equations of motion, defined in terms of the influence coefficients, is then transformed into the travelling wave mode domain. This allows the equations of motion to be decoupled, and each travelling wave mode can be processed separately. This is achieved by expanding the influence coefficients over the inter-blade phase angle domain by the discrete Fourier transform:

$$
C_{\sigma}=\sum_{n=0}^{N-1} L_{n} e^{-i n \sigma}
$$

where $\sigma$ is the inter-blade phase angle (IBPA), which, for the vibration in nodal diameter $N D$, is given by $\sigma=2 \pi N D / N$. Here, positive IBPAs correspond to forward travelling waves, and negative IBPAs to backward travelling waves. When the equation of motion is written in terms of travelling wave mode coefficients, the eigenvalues $\lambda_{\sigma}$ can be computed to determine the stability of the aeroelastic system at each inter-blade phase angle (IBPA). The aerodynamic damping ratio is given by $\zeta=-\operatorname{Re}\left(\lambda_{\sigma}\right) / \operatorname{Im}\left(\lambda_{\sigma}\right)$, where a negative damping ratio indicates that the modal force is in phase with the modal velocity, causing work to be exerted on the blade, resulting in flutter. Unless otherwise indicated, aerodynamic damping is measured as the aerodynamic damping ratio in this paper.

\section{Validation of CFD}

\subsection{Steady State Flow}

Before the parameters that drive flutter are investigated, the steady RANS CFD results are compared against time-averaged experimental measurements. It should be noted that in both cases, there is no blade vibration. Figure 8 shows CFD results for the pressure coefficient at nominal operating conditions. The blade loading is non-uniform as a pressure gradient exists across the cascade, as seen by the negative pressure gradient from blade +5 to -5 in Figure 8 . This is caused by the straight endwalls which prevent uniform flow deflection, resulting in a higher mean static pressure and a higher lift on Blade +5 than Blade -5. Separate CFD simulations (not included here) showed that this nonuniformity has a negligible effect on unsteady pressure distributions and the aerodynamic damping procedure.

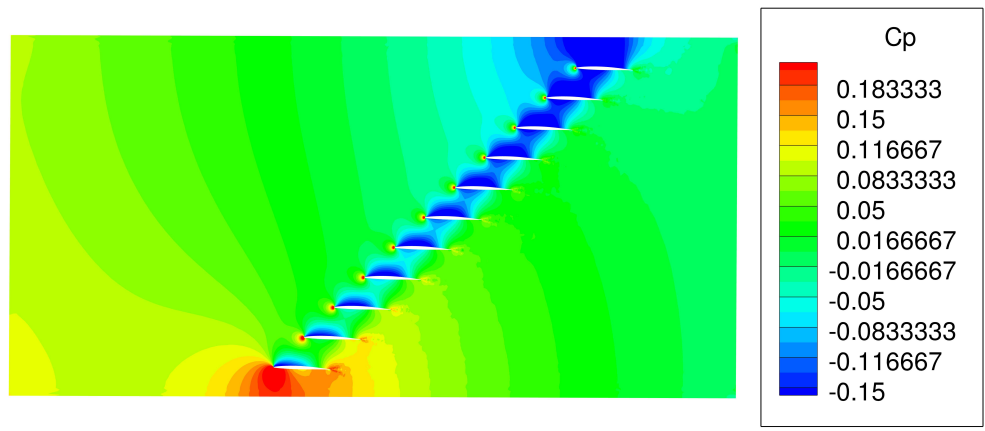

Figure 8. Computed normalised mid-span steady pressure contour.

Figure 9 shows the validation of the steady mid-span blade surface pressure coefficients as a function of normalised chord $l / c$. There is a generally good agreement over the three central blades, particularly towards the trailing edge. Towards the leading edge, there are some minor differences in blade loading. Possible explanations for this are the differences in incidence between the measurement and the CFD. The non-uniformity in loading can also be seen as blade +1 having a considerably larger pressure coefficient than blade -1 . 


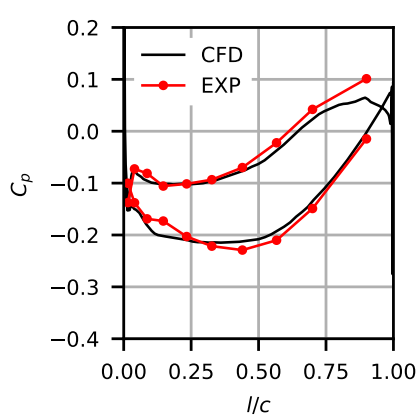

(a) blade +1

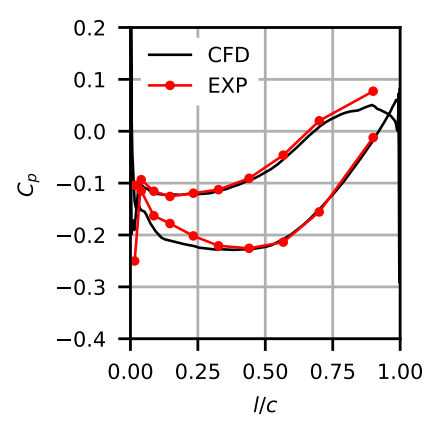

(b) blade 0

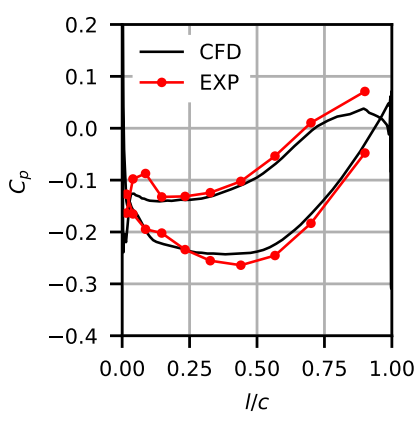

(c) blade -1

Figure 9. Validation of the steady mid-span surface pressure coefficient against normalised chord.

\subsection{Unsteady Flow}

To validate the unsteady CFD, the unsteady pressure on the blade surfaces are compared against experimental data. The pressure signals are Fourier-decomposed into the amplitude and phase at the blade vibration frequency. The results are shown in terms of the unsteady pressure coefficient in Figure 10 for $k=0.4$ and $x / c=1$ across the five central blades. The phase is shown with respect to the modal velocity. Due to the subsonic attached flow, the Fourier components in frequencies not equal to the vibration frequency are negligible. The amplitudes of unsteady $C_{p}$ from CFD are well-predicted, with a total error of approximately $9 \%$ across all blades shown. The largest amplitudes occur at the leading edge of the vibrating blade 0 . The unsteady disturbance from blade 0 propagates towards blade -1 , causing a large unsteady pressure amplitude on the pressure side with a very small change to the suction side. On the other hand, for blade +1 , there is a weaker propagating disturbance which impinges on the suction side, causing much large amplitudes compared to the pressure side of the blade. We can also see that this propagation mechanism is consistent with blades +2 and -2 as the shape of the unsteady pressure amplitudes and phases are comparable. There is very little phase change from the leading edge pressure side of blade 0 to the trailing edge of blade +1 on the suction side. A similar trend is seen on blade -1 .

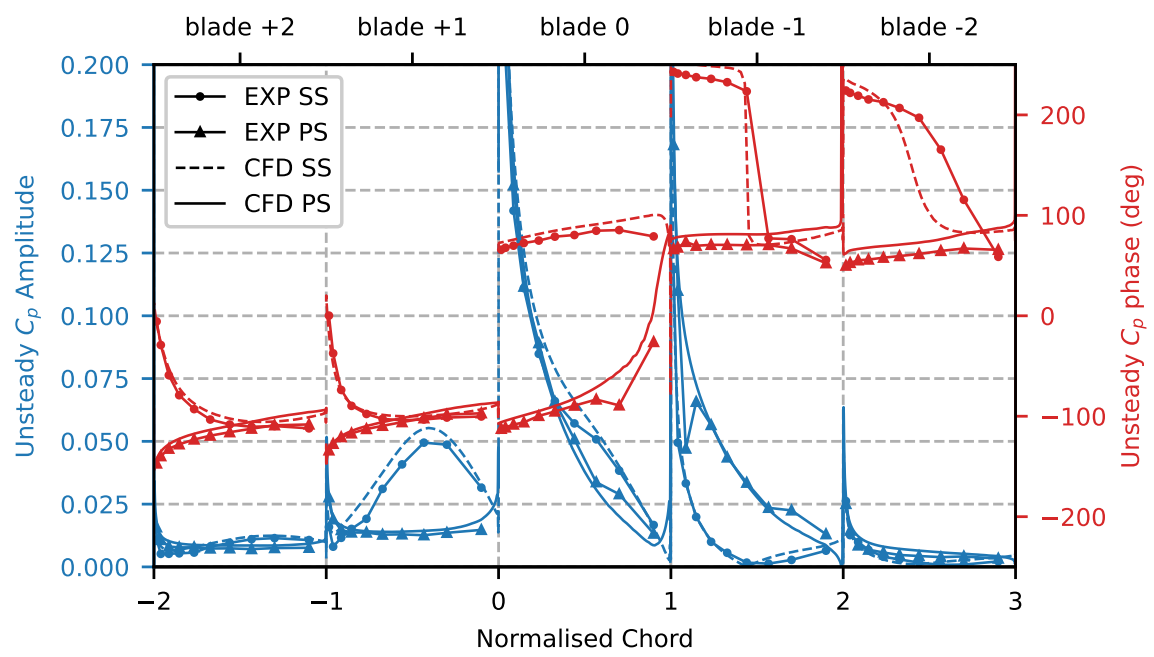

Figure 10. Validation of the unsteady mid-span blade surface pressure coefficient $-k=0.4, x / c=1$, $\phi=0.2$.

This indicates a nearly instantaneous propagation of forcing from blade 0 to the adjacent blades, which implies that it is the passage area change which drives the propagation. 
The integrated modal force and displacement for each blade are converted into the aerodynamic influence coefficients to begin the aerodamping analysis. Figure 11 shows the validation of the complex influence coefficients for blades +3 up to -3 . Coefficients with a positive imaginary component will cause instability as they are in phase with the modal velocity, causing work to be exerted on the blade by the flow. It can be seen that blade 0 provides a large stabilising contribution. In all tested modes, blade 0 always has a stabilising contribution as the forcing is damped by its own vibration [16]. Interestingly, all minus blade AICs for this particular mode also produce a stabilising contribution as the forcing is lagging the vibration. We can see from Equation (3) that blades with a greater discrete distance from the vibrating blade 0 will have considerably smaller contributions to the travelling wave mode coefficients. We can also see from Figure 11 that the AICs of the blades furthest away $(+3$ and -3$)$ themselves have a much smaller magnitude. For this reason, coefficients for blades $(> \pm 3$ ) will be neglected when calculating the aerodamping. This also allows for consistency with the experimental analysis. The aerodynamic damping ratio can now be calculated by the eigenanalysis shown in Section 5. Figure 12 shows the validation of aerodamping against the inter-blade phase angle. There is good agreement in terms of the shape of the aerodamping curve at each IBPA; however, the minimum aerodamping is under-predicted by approximately $30 \%$. Overall, these results show that CFD correctly predicts the operating condition and aerodamping and can therefore be used to continue with further stability analysis.

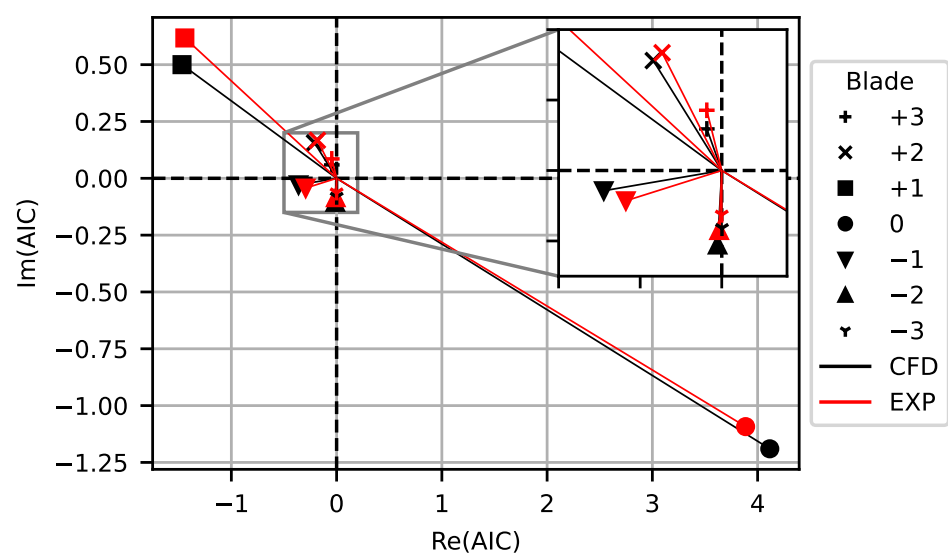

Figure 11. Complex AIC validation- $k=0.4, x / c=1, \phi=0.2$.

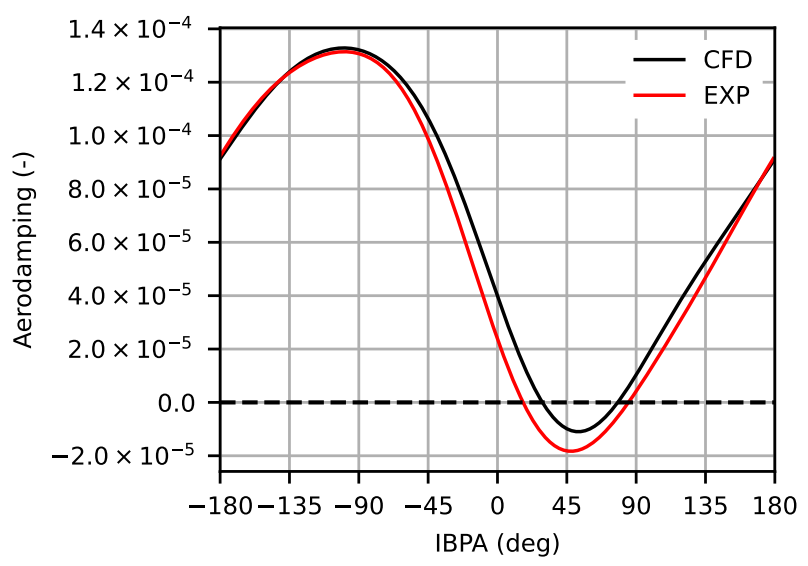

Figure 12. Aerodynamic damping ratio validation $-k=0.4, x / c=1, \phi=0.2$. 


\section{Stability Analysis}

Following the successful validation of the aerodamping for selected operating conditions, the numerical simulations are used to predict the aerodynamic damping over a wider range of reduced frequencies, $k$, and normalised pitching axis locations, $x / c$. The reduced frequencies are varied between 0.2 and 0.7 , and the torsion axis is varied between 0.5 and 3, where the former corresponds to a pure twist mode and the latter contains a large plunge component.

\subsection{Influence of Inter-Blade Phase Angle}

Figure 13 shows the curves of aerodynamic damping against the inter-blade phase angle (IBPA) for two different reduced frequencies, $k=0.2$ and $k=0.6$. While negative nodal diameters are stable for the whole range of frequencies and pitching axes, low ND forward travelling wave modes reach negative damping and instability. This is typically expected for $1 \mathrm{~F}$ flutter of this kind. The largest range of unstable IBPAs exists for low reduced frequencies and $x / c=0.5$. This pure torsion mode shape is unstable between 10 and $160^{\circ}$ IBPA at the lowest reduced frequency, $k=0.2$. The plunge-dominated mode, $x / c=3$, has a very narrow unstable IBPA range at the same frequency. Interestingly, the minimum aerodamping at $k=0.2$ does not occur at $x / c=0.5$ but is instead at $x / c=1.25$. This indicates that the mode stabilises as $x / c$ tends to 0.5 for a pure torsional mode shape at this frequency. With increasing frequency, the unstable region shrinks in IBPA range and in aerodamping magnitude, until only $x / c=0.5$ and $x / c=0.7$ are marginally unstable at $k=0.6$.

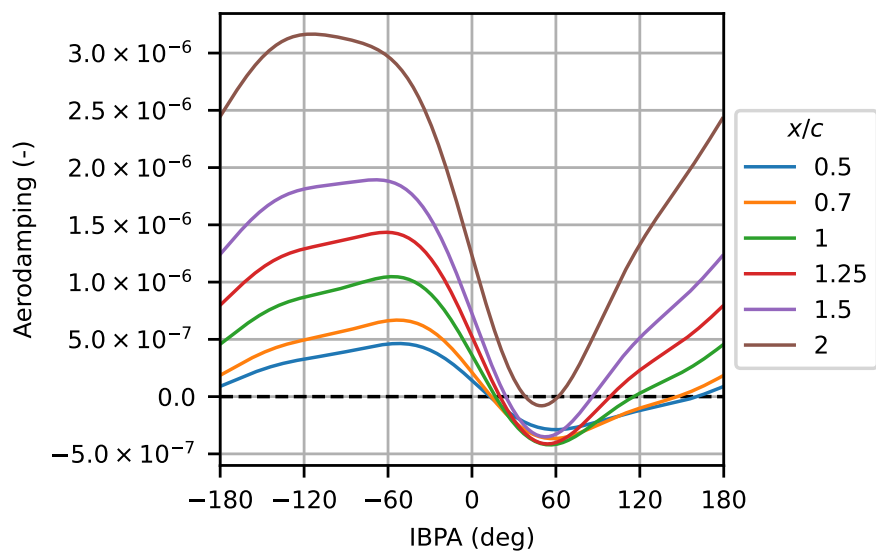

(a) $k=0.2$

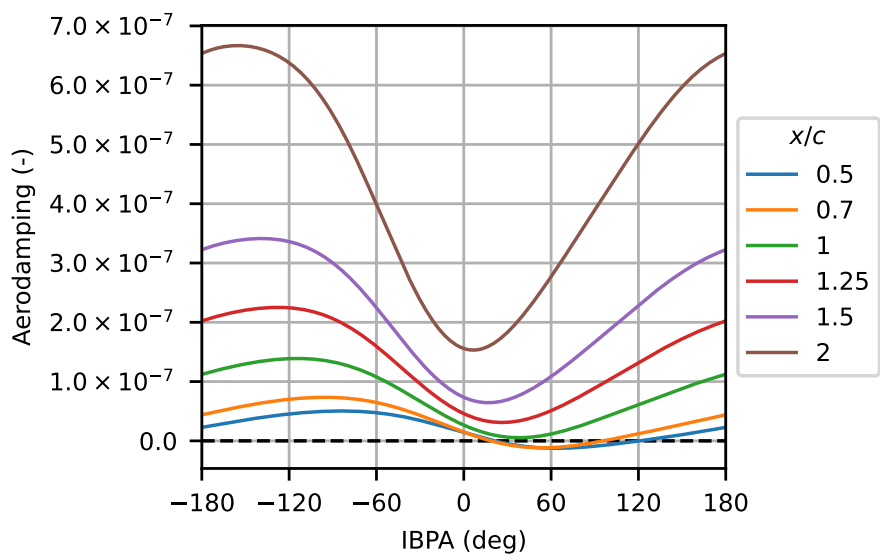

(b) $k=0.6$

Figure 13. Aerodamping as a function of the inter-blade phase angle. 
The aerodamping curves at $k=0.2$ and $k=0.6$ are significantly different. By looking at Equation (3), we can see that the travelling wave modes are harmonics of a Fourier series, where each coefficient is represented by pairs of blades a greater discrete distance from blade 0 . Blade 0 corresponds to the zeroth Fourier coefficient, which will be constant and positive over all IBPAs. The inclusion of the two nearest neighbours in the calculation of the travelling wave mode adds another harmonic and so on. Figure 14 shows how such harmonics are summated to form the aerodamping. We can see from $k=0.2$ in Figure $13 \mathrm{a}$ that the higher order harmonics contribute significantly to the aerodamping compared to $k=0.6$ in Figure $13 b$, which has a near cosine shape at $x / c=2$. We can therefore conclude that at low reduced frequencies, unsteady disturbances propagate more effectively to the blades that are further away as compared to higher frequencies, which in turn decrease aerodamping.

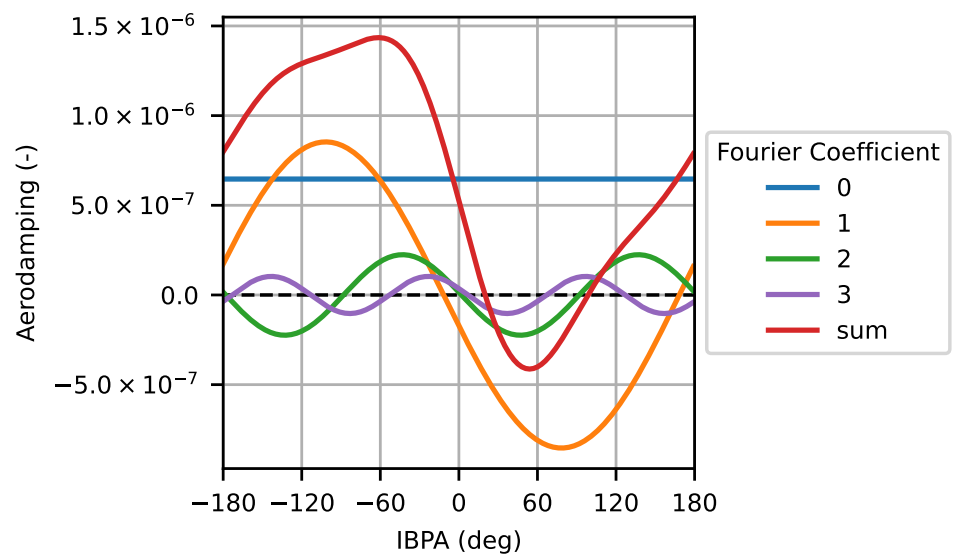

Figure 14. Aerodamping Fourier harmonics against IBPA- $k=0.2, x / c=1.25$.

\subsection{Influence of Reduced Frequency}

To investigate these trends further, aerodynamic damping is plotted as a function of reduced frequency for the different values of $x / c$ at a constant IBPA. This is shown in Figure 15. An IBPA of $50^{\circ}$ was selected because this was one of the least damped for the whole range of frequencies. Comparing the curves in Figure 15, it is clear that the reduced frequency has a strong influence on the aerodynamic damping trends. However, different trends are seen across the range of torsion axis locations. When the torsion axis is inboard of the aerofoil, $x / c \leq 1$, aerodynamic damping is negative for all reduced frequencies and asymptotically tends to zero with increasing frequency. The magnitude of aerodamping increases as the torsion axis shifts from the centre of the chord $(x / c=0.5)$ to the trailing edge $(x / c=1)$. Mode shapes with a larger plunge component, $1.5 \leq x / c \leq 3$, reach a local maximum at some reduced frequency, typically in the region of $k=0.25$. These curves then asymptotically tend to zero at greater reduced frequencies as the blade becomes too stiff to be affected by the flow. Additionally, the higher the plunge component, the lower the critical reduced frequency required to stabilise the blade. 


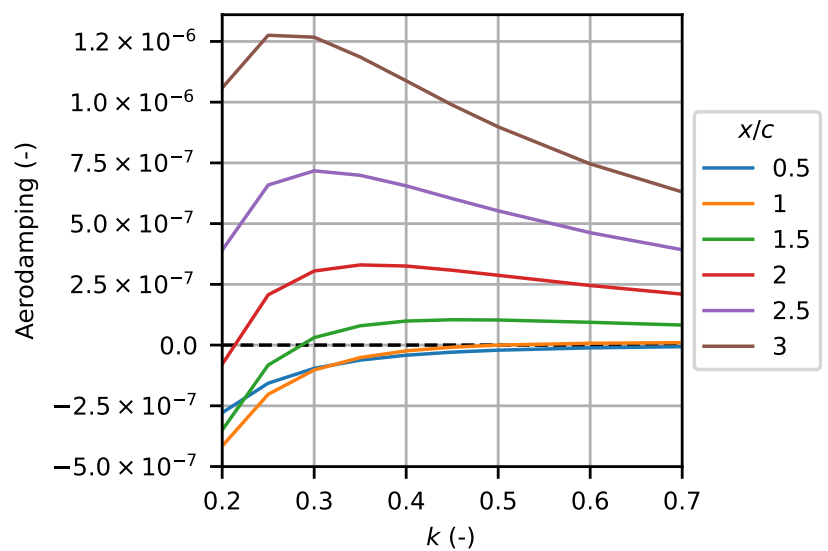

Figure 15. Effect of $k$ on aerodamping at constant $x / c-\mathrm{IBPA}=50^{\circ}$.

\subsection{Influence of Torsion Axis Location}

To further illustrate the influence of the torsional axis setback, Figure 16 shows the trend of aerodamping as the torsion axis setback is increased while reduced frequency and IBPA are kept constant. The modes are negatively damped for low $x / c$, and the curves follow a parabolic trend, with the critical $x / c$ decreasing for higher frequencies. For a reduced frequency of $k=0.2$, for example, the rotor is stable for $x / c>3$, whereas $k=0.4$ becomes stable at $x / c=2$. In other words, a higher torsional component leads to a larger range of unstable reduced frequencies. The minimum aerodamping does not occur at the lowest value of $x / c$, indicating that a pure torsion mode is more stable than a combined mode. This is expected from the literature as it is the cross-coupling of forcing from the plunge and twist motion which causes the greatest instability. We see that there are no monotonic trends with $x / c$ alone, but that it depends on frequency; hence, a stability parameter must contain a combination of the frequency and the plunge-to-twist ratio. From this and Equation (1), it can be deduced that the stability boundary for this IBPA is at a plunge-to-twist incidence ratio of approximately $\phi=0.27$.

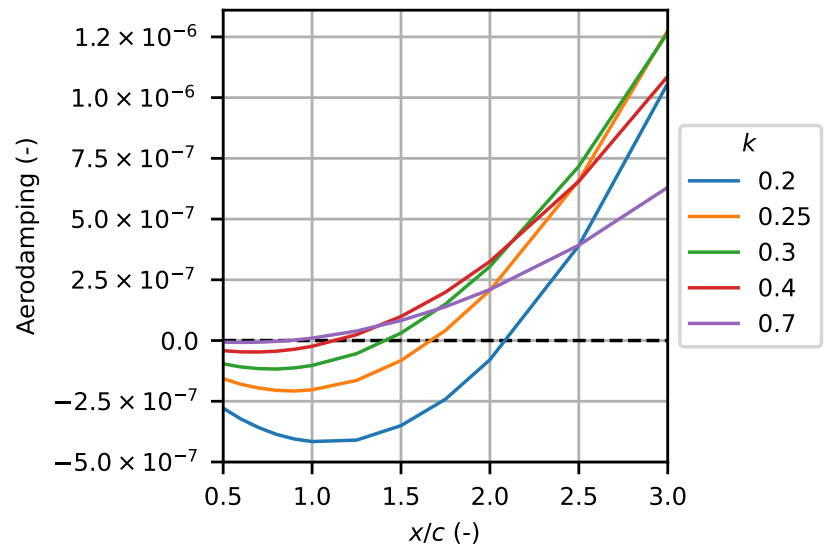

Figure 16. Effect of $x / c$ on aerodamping at constant $\mathrm{k}-\mathrm{IBPA}=50^{\circ}$.

\subsection{Stability Map}

To illustrate the behaviour of the whole range of inter-blade phase angles, the minimum aerodynamic damping over all inter-blade phase angles is extracted for each combination of reduced frequency and torsion axis setback. The resulting stability map is shown in Figure 17. As already indicated above, the stability boundary lies at an approximately constant incidence ratio of $\phi=0.27$, indicating the relevance of the plunge-to-twist incidence ratio parameter. We can see that this is slightly below the experimentally determined 
limit of $\phi=0.3$, indicating an error in the CFD of approximately $10 \%$. The magnitude of the minimum aerodynamic damping does not scale with the incidence ratio, as the isolines do not follow lines of constant $\phi$. Instead, there is a region of very large negative aerodamping for mode shapes with a large torsional component at low reduced frequencies. The maximum aerodamping for the parameter range tested is in the plunge-dominated mode, $x / c=3$, and a reduced frequency of $k=0.25$. Interestingly, it is not the largest reduced frequency which has the largest positive aerodamping, indicating that blade stiffness could be sacrificed to obtain a larger $x / c$.

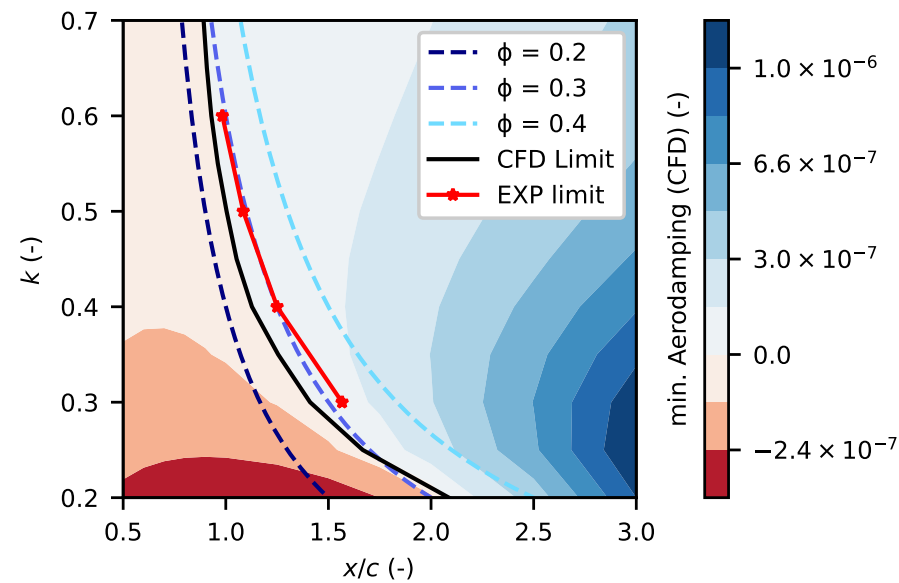

Figure 17. Stability map with contours of minimum aerodamping (CFD) as a function of $k$ and $x / c$.

\subsection{Discussion}

Comparing these results to Whitehead's theoretical study of flat plates in an infinite cascade, we see some similarities. Figure 18 plots the critical reduced frequency, below which the cascade becomes unstable. The cascade geometry is similar to the one investigated here, with the same stagger angle but a larger pitch-to-chord ratio $(s / c=1$ instead of $s / c=0.75)$. The curve of interest for this paper is the incompressible limit, $M=0$. The unstable region predicted by Whitehead is very similar to Figure 17 for the reduced frequency of $k=0.7$, for example, which becomes unstable at approximately $x / c=1$. However, this is not consistent at lower reduced frequencies. This can be partially attributed to the different cascade geometry, since parametric investigations by Whithead [1] showed that reducing the pitch-to-chord ratio will extend the unstable range. However, other differences also exist. The blades in this study have a finite camber, steady loading, and viscous effects that are considered. The stability boundary predicted here also differs from that of transonic fans. According to the work by Vahdati and Cumpsty [3] and Lee et al. [8], the stability boundary for transonic fans near stall lies at $\phi \approx 1$. However, their research also showed a strong dependence of stability on steady state aerodynamics. The importance of these effects, and how they can be built into stability parameters, will be the subject of future work. 


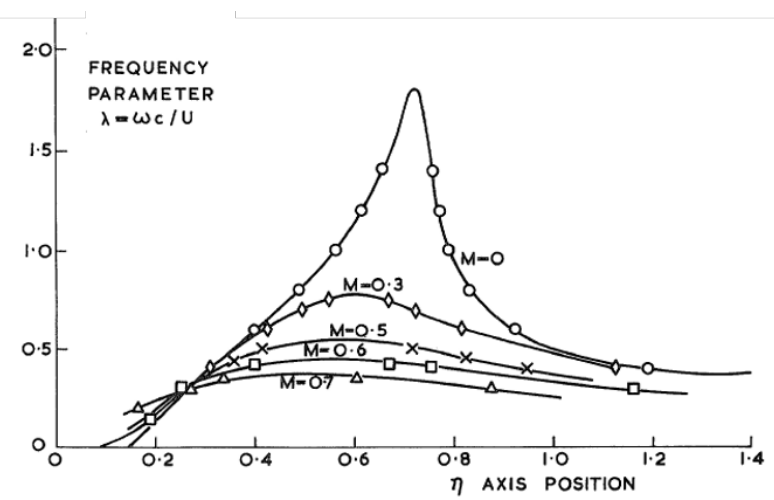

Figure 18. Stability map for an infinite cascade of flat plates with $\xi=45^{\circ}, s / c=1.0, \eta=x / c$. (Whitehead, 1974).

\section{Conclusions}

This paper investigated the influence of pitching axis location and reduced frequency on the aeroelastic stability of a subsonic, unstalled, linear compressor cascade. Numerical simulations were validated against experimental measurements and consequently used for a parametric study. The range of reduced frequencies and mode shapes tested are representative of those found in modern compressors, but owing to the low incidence angle, low camber, and subsonic inflow, the cascade loading is comparatively low. This is one possible reason why the stability limit occurs at much lower incidence ratios compared to the literature for highly loaded fan blades. The results confirmed the strong dependency of stability on the reduced frequency and the plunge-to-twist component reported in the literature. We also showed that there are no monotonic trends between these parameters, hence a stability parameter must contain a combination of frequency and the plunge-totwist ratio. It was also shown that the minimum aerodamping does not occur at a pure twist mode shape, but that it is rather a combination of plunge and twist forcing, which creates the largest instability when $x / c$ is aft of half chord. The ratio of the incidence induced by the plunge motion to that induced by the twist motion was shown to be a valid stability criteria for early engine design stages, despite the changes in loading compared to a transonic fan. In further studies, the effects of blade loading and inlet Mach number will be considered.

Author Contributions: Conceptualization, S.S. (Sabine Schneider), D.P. and S.S. (Sina Stapelfeldt) data curation, G.H. and J.G.; formal analysis, G.H. and J.G.; funding acquisition, S.S. (Sabine Schneider), D.P. and S.S. (Sina Stapelfeldt); investigation, G.H. and J.G.; methodology, G.H. and J.G.; project administration, S.S. (Sabine Schneider); software, G.H. and J.G.; supervision, D.P. and S.S. (Sina Stapelfeldt); visualization, G.H.; writing — original draft, G.H. and S.S. (Sina Stapelfeldt); writingreview and editing, G.H., J.G., S.S. (Sabine Schneider), D.P. and S.S. (Sina Stapelfeldt). All authors have read and agreed to the published version of the manuscript.

Funding: This research was funded by an industrial collective research programme (IGF/CORNET no. 128 EN, FVV Project Number 1331). It was supported by the Federal Ministry for Economic Affairs and Energy (BMWi) through the AiF (German Federation of Industrial Research Associations $\mathrm{eV}$ ) and the Engineering and Physical Science Research Council through an iCASE studentship (Ref 18000181).

Institutional Review Board Statement: Not applicable.

Informed Consent Statement: Not applicable.

Data Availability Statement: Not applicable.

Acknowledgments: The authors would like to thank Rolls-Royce plc for sponsoring this study and allowing its publication. The authors additionally thank Bernhard Mueck of Rolls-Royce Deutschland and Caetano Peng of Rolls-Royce UK for their scientific input. 
Conflicts of Interest: The authors declare no conflict of interest.

\section{Nomenclature}

The following nomenclature is used in this manuscript:

$\begin{array}{ll}\text { 1F } & \text { First Flap Blade Mode } \\ \text { AIC } & \text { Aerodynamic Influence Coefficient } \\ \text { IBPA } & \text { Inter Blade Phase Angle } \\ \text { ND } & \text { Nodal Diameter } \\ \text { RANS } & \text { Reynolds Averaged Navier-Stokes } \\ \text { SDOF } & \text { Single Degree of Freedom } \\ \text { PS } & \text { Pressure Side } \\ \text { SS } & \text { Suction Side } \\ \text { VBM } & \text { Vibrating Blade Module } \\ \zeta & \text { Aerodynamic Damping Ratio } \\ \theta_{\text {plunge }} & \text { Plunge Incidence Amplitude } \\ \theta_{\text {pitch }} & \text { Pitch Incidence Amplitude } \\ \xi & \text { Stagger Angle } \\ \phi & \text { Plunge-to-Pitch Incidence Ratio } \\ c & \text { Chord } \\ f & \text { Vibration Frequency } \\ k & \text { Reduced frequency, } k=2 \pi f c / u \\ M & \text { Mach Number } \\ s & \text { Pitch } \\ u & \text { Inlet Flow Velocity } \\ x & \text { Pitch Axis Location } \\ z & \text { Lever Length } \\ & \end{array}$

\section{References}

1. Whitehead, D.S. The Effect of Compressibility on Unstalled Torsional Flutter. Aeronautical Research Council Reports \& Memoranda. 1974; Volume 3754. Available online: https:/ / reports.aerade.cranfield.ac.uk/handle/1826.2/3032 (accessed on 10 November 2021).

2. Halliwell, D. Fan Supersonic Flutter: Prediction and Test Analysis. Aeronautical Research Council Reports \& Memoranda. 1975; Volume 3789. Available online: https:/ / reports.aerade.cranfield.ac.uk/handle/1826.2/3068 (accessed on 5 November 2021).

3. Vahdati, M.; Cumpsty, N. Aeroelastic Instability in Transonic Fans. J. Eng. Gas Turbines Power 2015, 138, 022604. [CrossRef]

4. Panovsky, J.; Kielb, R.E. A Design Method to Prevent Low Pressure Turbine Blade Flutter. J. Eng. Gas Turbines Power 2000, 122, 89-98. [CrossRef]

5. Zhao, F. Impact of mode shape and acoustic gust on blade flutter stability. In Proceedings of the 15th International Symposium on Unsteady Aerodynamics, Aeroacoustics \& Aeroelasticity of Turbomachines, ISUAAAT, Oxford, UK, 24-27 September 2018; pp. 1-10.

6. Whitehead, D.S. Force and Moment Coefficients for Vibrating Aerofoils in Cascade. Aeronautical Research Council Reports \& Memoranda. 1962; Volume 3254. Available online: https:/ / reports.aerade.cranfield.ac.uk/handle/1826.2/3828 (accessed on 20 November 2021).

7. Nipkau, J.; Power, B.; Jordan, M. Aeromechanical design and test of a modern highly loaded fan. In Proceedings of the ASME Turbo Expo 2017: Turbomachinery Technical Conference and Exposition, Charlotte, NC, USA, 26-30 June 2017; Volume 2, pp. 1-11. [CrossRef]

8. Lee, K.B.; Wilson, M.; Vahdati, M. Numerical study on aeroelastic instability for a low-speed fan. J. Turbomach. 2017, 139, 071004 [CrossRef]

9. Carta, F.O. Unsteady Gapwise Periodicity of Oscillating Cascaded Airfoils. In Proceedings of the ASME 1982 International Gas Turbine Conference and Exhibit. American Society of Mechanical Engineers, London, UK, 18-22 April 1982; pp. 1-15. [CrossRef]

10. Malzacher, L.; Geist, S.; Peitsch, D.; Hennings, H. A Low Speed Compressor Test Rig for Flutter Investigations. In Proceedings of the Volume 7B: Structures and Dynamics, American Society of Mechanical Engineers, Seoul, Korea, 13-17 June 2016; pp. 1-12. [CrossRef]

11. Sayma, A.; Vahdati, M.; Imregun, M. An Integrated Nonlinear Approach for Turbomachinery Forced Response Prediction. Part 1: Formulation. J. Fluids Struct. 2000, 14, 87-101. [CrossRef]

12. Vahdati, M.; Simpson, G.; Imregun, M. Mechanisms for wide-chord fan blade flutter. J. Turbomach. 2011, 133, 041029. [CrossRef]

13. Stapelfeldt, S.; Vahdati, M. On the importance of engine- representative models for fan flutter predictions. J. Turbomach. 2018, 140, 081005. [CrossRef] 
14. Lee, K.B.; Wilson, M.; Vahdati, M. Validation of a numerical model for predicting stalled flows in a low-speed fan-Part I: Modification of Spalart-Allmaras turbulence model. J. Turbomach. 2018, 140, 051008. [CrossRef]

15. Hanamura, Y.; Tanaka, H.; Yamaguchi, K. A Simplified Method to Measure Unsteady Forces Acting on the Vibrating Blades in Cascade. Bull. JSME 1980, 23, 880-887. [CrossRef]

16. Crawley, E.F.; Hall, K.C. Optimization and mechanisms of mistuning in cascades. J. Eng. Gas Turbines Power 1985, 107, 418-426. [CrossRef] 